\title{
12) Efeito de intervenções cognitivas na autoeficácia e na memória episódica de idosas do Distrito Federal
}

\author{
Angela Maria Sacramento'; Cristiane Holanda Costa²; João Lucas Araujo Assunção²; \\ Caio Diogo Santana de Sousa²; Fernanda de Sousa²; \\ Danilo Ribeiro do Nascimento Aragão²; Amanda Alves da Silva \\ Roberto Menezes de Oliveira ${ }^{4}$; Virgínia Turra ${ }^{4}$; Gislane Ferreira de Melo \\ Karla Helena Coelho Vilaça e Silva ${ }^{4}$; Maria Liz Cunha de Oliveira ${ }^{4}$; \\ Henrique Salmazo da Silva ${ }^{4}$; Isabelle Patriciá Freitas Soares Chariglione ${ }^{4}$
}

\section{Resumo}

As intervenções cognitivas são utilizadas para aprimoramento de funções cognitivas e diversos estudos têm apresentado correlações entre medidas de autoeficácia e cognitivas. Esse estudo buscou verificar o desempenho de idosas em tarefas de memória episódica verbal e na percepção subjetiva em medidas de autoeficácia geral. O delineamento foi do tipo quantitativo, experimental e transversal, sendo avaliadas 39 idosas com média de idade igual a 67,77 (DP $\pm 6,34$ ), em medidas cognitivas e psicológicas em dois momentos. Para a análise dos dados foram utilizados testes não-paramétricos (Spearman e Wilcoxon) que indicaram correlações com o RAVLT recordação A6 (0,431, p=0,006); e com o GDS (-0,376, p=0,016), e as análises de pré e pós-treino indicaram que as participantes apresentaram ganhos no desempenho de algumas funções cognitivas. Concluindo-se que as intervenções cognitivas foram promissoras nas idosas investigadas e que apresentaram impacto em medidas de memória objetivas e subjetivas.

Palavras-chave: autoeficácia, idoso, cognição; treino, memória.

1 Doutoranda no Programa de Pós Graduação Stricto Sensu em Gerontologia da Universidade Católica de Brasília, Campus I, Pesquisadora no Projeto NeuroCog-Idoso. Endereço para correspondência: QS 7, Lote 1 EPCT Sala 009, Águas Claras, Brasília, DF, 71966-700. Email: sacrapesquisa@gmail.com

2 Graduandos em Psicologia, Pesquisadora no Projeto NeuroCog-Idoso. Emails (na ordem em que aparecem): cristianeholandacc@gmail.com; joao.luq@gmail.com; caio.psic23@gmail.com; mrsfernandarocha@gmail.com; daniloraragao@hotmail.com;

3 Doutoranda no Programa de Pós Graduação Stricto Sensu em Gerontologia da Universidade Católica de Brasília. Email: amandaalvesgerontologia@gmail.com

4 Professores e Pesquisadores no Projeto NeuroCog-Idoso. Email (na ordem em que aparecem): roberto.bob.df@gmail. com; turra.virginia@gmail.com; gmelo@p.ucb.br; kavilaca@yahoo.com.br; lizcunhad@gmail.com; henriquesalmazo@ gmail.com; isabelle.chariglione@p.ucb.br 


\section{Intervenções cognitivas na autoeficácia e na memória episódica de idosas}

O envelhecimento cursa com um gradual declínio das funções cognitivas, sendo o declínio da memória a que mais se destaca entre as principais queixas da população em geral, na qual compromete as tarefas relativas às atividades instrumentais da vida diária (MELO et al, 2017; BELLEVILLE et al, 2018). As maiores queixas relacionam-se à memória de trabalho, principalmente na redução dos recursos de processamento, e às falhas no mecanismo de inibição de informações supérfluas (BOLLER et al, 2017). Por consequência, o processamento da informação ocorre de forma mais lentificada, na qual há uma competição das informações necessárias com as irrelevantes (WEST et al, 2009).

Na relação do indivíduo e o ambiente, destaca-se a autoeficácia, que pode ser definida como a crença que o indivíduo tem sobre sua capacidade de realizar, com sucesso, determinada atividade, e orienta a ação de uma pessoa sobre si mesma e sobre o ambiente (BANDURA et al, 2009). Para Bandura et al (2009) julgamentos sobre as competências alheias são construções sociais que servem para classificar os indivíduos em estereótipos e estigmas. Por exemplo, a relação entre declínio de memória e avanço da idade é um estigma social intimamente relacionado com o idoso (CARNEIRO; FALCONE, 2013).

Segundo Neri (2006), os indivíduos criam suas expectativas de acordo com suas crenças e, neste contexto, consolidam paradigmas sobre a velhice, como do idoso perder competência, e passam a se comportar conforme suas crenças. Portanto, percebe-se que crenças de autoeficácia influenciam o modo como às pessoas sentem, pensam e agem.

Diversos estudos têm destacado o papel da autoeficácia com o desempenho objetivo de memória, e em especial, a possível contribuição das intervenções cognitivas no desempenho objetivo e subjetivo de memória. Contudo, a relação entre essas variáveis necessita ser melhor explorada na população de idosos brasileira em função das características sociodemográficas e culturais (YASSUDA et al, 2005; MAGALHÃES; HAMDAN, 2010). Assim, este estudo teve como objetivo verificar o desempenho de idosas do Distrito Federal em tarefas de memória episódica verbal e na percepção subjetiva da autoeficácia geral e da memória.

\section{Materiais e Métodos}

O estudo foi desenvolvido na Universidade Católica de Brasília, no Grupo de Pesquisa em Neurociência e Cognição - Idoso (NeuroCog-Idoso). O delineamento caracterizou-se por uma pesquisa quantitativa, experimental e transversal.

\section{Amostra}

A amostra que iniciou nos programas de treino e estimulação foi composta por $44 \mathrm{mu}-$ lheres idosas. Destas participantes, 39 finalizaram as intervenções e tiveram frequência igual ou superior a $75 \%$ nas sessões propostas, sendo essa a amostra investigada. A média de idade da amostra foi de 67,77 $\pm 6,34$ anos (60-87), e 20 participantes tinham escolaridade até o ensino fundamental completo e 19 entre o ensino médio ao ensino superior completo. O presente estudo foi submetido ao CEP da Universidade Católica de Brasília e aprovado com o CAAE: 67653517.4.0000.0029. 


\section{Instrumentos}

Para avaliação da memória, foi utilizado o Teste de Aprendizagem Auditivo-Verbal de Rey (RAVLT), teste de medida breve, que acessa a capacidade de memória imediata (memória de trabalho - A1), o índice de aprendizagem (somatória de A1 a A5), a evocação tardia livre (memória longo prazo - A7) e a capacidade de recordação (REC) (MAGALHÃES; HAMDAN, 2010; MALLOY-DINIZ et al, 2007). A versão utilizada no presente estudo é a mesma do estudo de Lima (2007), sendo que a pontuação máxima das variáveis é de 15, exceto o índice de aprendizagem (A1 a A5), que é de 75.

Para a mensuração da autoeficácia, foram utilizados o Teste de Percepção Subjetiva da Memória (MAC-Q) e o Questionário de Memória Prospectiva e Retrospectiva (PRMQ-10). O MAC-Q é um instrumento de autoadministração, com objetivo de investigar como o indivíduo percebe a competência da sua memória. Com pontuação máxima de 35 pontos, neste teste quanto maior o valor, mais negativa é a percepção de falha da memória (MATTOS et al, 2003). O teste PRMQ-10 objetivou avaliar o autorrelato de falhas de memória prospectiva e retrospectiva. $\mathrm{O}$ valor máximo e mínimo é de 50 e 10 pontos, respectivamente, com ponto de corte 20 , visto que os indivíduos que apresentam pontuação igual ou acima de 20 apresentam alta queixa de falha de memória (BENITES; GOMES, 2007). Nesse estudo considerou-se a pontuação total.

Adicionalmente os participantes foram avaliados pelo Geriatric Depression Screening scale - escala de depressão geriátrica - GDS - instrumento de 15 itens adaptado para a população brasileira por Almeida e Almeida (1999); Escala de Ansiedade de Beck (CUNHA, 2011) composto por 21 questões de múltipla escolha, cujo escore variará de 0 a 63 pontos para medidas de ansiedade, e a Escala de Auto Eficácia Geral (SBICIGO et al, 2012) que trata-se da versão de validação do instrumento para a população brasileira e que apresenta características psicométricas adequadas e o sujeito responde a cada item por quatro pontos: 1 = "Não é verdade a meu respeito"; 2 = "É dificilmente verdade a meu respeito"; 3 = "É moderadamente verdade a meu respeito"; e 4 = "É totalmente verdade a meu respeito". O total da escala resulta da soma dos valores de todos os itens. Quanto maior o valor, maior é a autoeficácia geral percebida.

\section{Procedimento metodológico}

Tendo em vista o delineamento para o recrutamento dos participantes idosos, inicialmente foi realizada uma palestra sobre Envelhecimento, Cognição e Atividade Física, que finalizava com a explanação da pesquisa e convite aos idosos para participar do grupo de pesquisa NeuroCog- Idoso. Após o convite e aceite do mesmo, houve contato posterior para marcar a avaliação inicial, com duração aproximada de 60 minutos para cada idoso.

No segundo momento, o da intervenção cognitiva, os idosos foram divididos em dois grupos (Stimullus e MEMO), com encontro semanal, por seis semanas, com duração de 90 minutos cada sessão, ao término todos os idosos foram reavaliados.

A sequência de aplicação dos instrumentos seguiu uma ordem, que compreendeu a leitura do TCLE, o preenchimento do questionário sociodemográfico, a aplicação do instrumento 
Lista de Aprendizagem Auditivo-Verbal de Rey, seguido pelo teste de percepção subjetiva da memória e o Questionário de Memória Prospectiva e Retrospectiva.

\section{Procedimento de análises de dados}

Os dados foram analisados através do estatístico Statistical Package for Social Science (SPSS) (versão 22.0), devidamente registrado para o projeto. Inicialmente observou-se que os dados não seguiam uma distribuição normal, e por esta razão optou-se pelos testes não-paramétricos de Spearman para verificar correlação entre as medidas investigadas no pré-teste e pelo teste não-paramétrico para comparação de medidas repetidas pré e pós-treino de Wilcoxon. O nível de significância adotado foi de $\mathrm{p} \leq 0,05$.

\section{Resultados/Conclusões}

As análises de correlações bivariadas de Spearman indicaram que na linha de base a Auto Eficácia Geral (AEG) apresentou correlações com o RAVLT recordação A6 (0,431, $\mathrm{p}=0,006)$; e com sintomas depressivos no $\operatorname{GDS}(-0,376, \mathrm{p}=0,016)$, indicando que quanto maior a autoeficácia geral melhor o desempenho de memória episódica e menor a prevalência de sintomas depressivos. Houve também correlação entre a MAC Total e a Escala de Ansiedade de Beck $(0,461, p=0,003)$, sugerindo que quanto mais negativa é a percepção de falha da memória maior é a pontuação na escala de ansiedade.

As análises de pré e pós-treino indicaram que as participantes apresentaram ganhos no desempenho de memória episódica expressos nos testes RAVLT recordação A6, recordação A7 e Curva de aprendizagem; e melhor Auto Eficácia Geral. Observou-se tendência de redução dos sintomas depressivos e aumento do índice de Recuperação (relação recordação A6/A5). Não houve alterações nas demais medidas investigadas.

Tabela 1. Pré e pós-intervenção de mulheres idosas participantes dos grupos de programa intervenção cognitiva MEMO e Stimullus $(\mathrm{n}=39)$

\begin{tabular}{|c|c|c|c|}
\hline & $\begin{array}{l}\text { Pré-treino } \\
\text { Média (DP) }\end{array}$ & $\begin{array}{c}\text { Pós-treino } \\
\text { M (DP) }\end{array}$ & $P$ valor \\
\hline RAVLT Lista B & $4,46(+2,25)$ & $4,79(+1,80)$ & 0,155 \\
\hline RAVLT Recordação A6 & $6,92(+2,85)$ & $8,56(+3,04)$ & 0,000 \\
\hline RAVLT Recordação A7 & $7,03(+2,78)$ & $8,64(+3,20)$ & 0,001 \\
\hline Curva de aprendizagem (A1/A5) & $39,13(+9,64)$ & $44(+10,44)$ & 0,001 \\
\hline $\mathrm{IP}(\mathrm{B} 1 / \mathrm{A} 1)$ & $1,15(+0,77)$ & $0,95(+0,51)$ & 0,174 \\
\hline IR (A6/A5) & $0,77(+0,42)$ & $0,90(+0,38)$ & 0,059 \\
\hline AEG Total & $32,44(+4,61)$ & $34,08(+4,48)$ & 0,025 \\
\hline MAC-Q Total & $26,72(+5,87)$ & $25,44(+6,48)$ & 0,140 \\
\hline PRMQ Total & $35,10(+8,69)$ & $36,74(+7,55)$ & 0,137 \\
\hline GDS & $4,23(+2,96)$ & $3,46(+3,04)$ & 0,059 \\
\hline Ansiedade Beck & $6,18(+7,24)$ & $7,64(+9,32)$ & 0,199 \\
\hline
\end{tabular}


Os achados do presente estudo indicam que as intervenções cognitivas geraram ganhos em medidas de memória episódica verbal e em teste de autoeficácia geral. Contudo, os ganhos não se aplicaram a medidas de autoeficácia de memória prospectiva e memória relacionada ao envelhecimento, o que pode indicar que os ganhos foram seletivos às estratégias de memória investigadas, voltadas para o processamento de informações visuais, categorização e habilidades mnemônicas gerais. Sendo assim, esperava-se que o treino em memória prospectiva pudesse eliciar ganhos no PRMQ, contudo, essa hipótese precisa ser melhor elucidada.

Outra questão refere-se a possível relação entre a autoeficácia, sintomas depressivos e desempenho de memória episódica. No pré-teste a AEG teve correlações tanto com o desempenho objetivo quanto com os sintomas depressivos. No pós-treino observou-se tendência de menor prevalência dos sintomas depressivos, indicando que a melhor autoeficácia geral pode ter sido mediada pela redução dos sintomas depressivos. Desse modo, resta saber, a interdependência entre os ganhos e se existem possíveis diferenças entre os grupos Stimullus e MEMO quanto aos desfechos observados. Em síntese as intervenções cognitivas foram promissoras nos idosos investigados e apresentaram impacto em medidas de memória objetivas e subjetivas que devem ser melhor explorados.

\title{
Reconhecimento e agradecimentos
}

A presente pesquisa foi realizada graças ao apoio financeiro $\mathrm{n}^{\mathrm{0}}: 0193.001227 / 2016 \mathrm{da}$ Fundação de Apoio à Pesquisa do Distrito Federal - FAPDF.

\section{The Effect of cognitive interventions on self-efficacy and episodic memory of elderly women in the Federal District}

\begin{abstract}
Cognitive interventions are used to improve cognitive functions and several studies have shown correlations between self-efficacy and cognitive measures. This study aimed to verify the performance of the elderly in tasks of verbal episodic memory and subjective perception in measures of general self - efficacy. A quantitative, experimental and cross - sectional design was used, with 39 elderly women with a mean age of 67.77 (SD \pm 6.34 ), in cognitive and psychological measures at two moments. For the data analysis we used non-parametric tests (Spearman and Wilcoxon) which indicated correlations with the AAV recall memory $(0,431$, $p=0.006)$; and GDS $(-0.376, p=0.016)$, and the pre and post-training analyzes indicated that the participants presented gains in the performance of some cognitive functions. It was concluded that the cognitive interventions were promising in the elderly women investigated and that they had an impact on objective and subjective memory measures.
\end{abstract}

Keywords: self-efficacy, elderly, cognition; training, memory. 


\section{Referências}

ALMEIDA, O. P. ALMEIDA, S. A. Confiabilidade da versão brasileira da Escala de Depressão em Geriatria (GDS) versão reduzida. Arquivos de Neuro-Psiquiatria, 1999, 57, 421-426.

BANDURA A, AZZI RG, POLYDORO SA. Teoria social cognitiva: conceitos básicos: Artmed Editora; 2009.

BELLEVILlE, S.; HUDSON, C., BIER, N.; BRODEUR, C.; GILBERT, B.; GRENIER, S.; OUELLET, M.C.; VISCOGLIOSI, C.; GAUTHIER, S. MEMO+: Efficacy, Durability and Effect of Cognitive Training and Psychosocial Intervention in Individuals with Mild Cognitive Impairment. Journal of the American Geriatrics Society. 2018;66(4):655-63.

BENITES, D., GOMES, W. B. Tradução, adaptação e validação preliminar do Prospective and Retrospective MEMOry Questionaire (PRMQ). Psico-USF, 2007, 12(1), 45-54. http://doi.org/10.1590/S1413-82712007000100006.

BOLLER B, MELLAH S, DUCHARME-LALIBERTÉ G, BELLEVILLE S. Relationships between years of education, regional grey matter volumes, and working MEMOry-related brain activity in healthy older adults. Brain imaging and behavior. 2017;11(2):304-17.

CARNEIRO RS, FALCONE E. O desenvolvimento das habilidades sociais em idosos e sua relação na satisfação com a vida. Estudos de psicologia. 2013;18(3):517-26.

CUNHA, J. A. Manual de português das Escalas Beck. São Paulo: Casa do Psicólogo, 2011.

LIMA, J.S. Efeitos do treino de memória e da atividade física em portadores da doença de Alzheimer. 2007, Dissertação (Mestrado em Psicologia), Universidade Federal de Santa Catarina, Florianópolis.

MAGALHÃES, S. S., \& HAMDAN, A. C. The Rey Auditory Verbal Learning Test: normative data for the Brazilian population and analysis of the influence of demographic variables. Psychology \& Neuroscience, 2010,3(1), 8591. doi: 10.3922/j.psns.2010.1.011.

MALLOY-DINIZ, L. F., LASMAR, V. A. P., GAZINELLI, L. S. R., FUENTES, D., SALGADO, J. V. The Rey Auditory-Verbal Learning test: applicability for the Brazilian erderly population. Rev. Bras Psiquiatria, 2007, 29(4), 324-329. http://dx.doi.org/10.1590/S1516-44462006005000053.

MATTOS, P., LINO, V., \& RIZO, L., ALFANO, A. ARAÚJO. K. RAGGIO, R. Queixas de memória de idosos saudáveis e desempenho em testes. Arquivos de Neuropsiquiatria, 2003, 61(4), 920-24. Recuperado de http://www. scielo.br/pdf/anp/v61n4/a06v61n4.pdf.

MELO, Laércio Almeida de; FERREIRA, Lidiane Maria de Brito Macedo; SANTOS, Marquiony Marques dos; LIMA, Kenio Costa de. Fatores socioeconômicos, demográficos e regionais associados ao envelhecimento populacional. Rev. bras. geriatr. gerontol., Rio de Janeiro, v. 20, n. 4, p. 493-501, ago. 2017. Disponível em <http:// www.scielo.br/scielo.php?script $=$ sci_arttext\&pid $=$ S1809-98232017000400493\&lng=pt\&nrm $=$ iso $>$. acessos em 08 jan. 2019. http://dx.doi.org/10.1590/1981-22562017020.170004.

NERI AL. O senso de auto-eficácia como mediador do envelhecimento bem sucedido no âmbito da cognição, das competências para a vida diária e do auto cuidado à saúde. Auto-eficácia em diferentes contextos. Campinas: Alínea. 2006:59-85.

SBICIGO, J.B., TEIXEIRA, M.A.P., DIAS, A.C.G., DELL `AGLIO, D.D. Propriedades psicométricas da escala de autoeficácia geral percebida (EAGP). PSICO, 2012, 43, 139-146.

WEST RL, DARK-FREUDEMAN A, BAGWELL DK. Goals-feedback conditions and episodic MEMOry: Mechanisms for MEMOry gains in older and younger adults. MEMOry. 2009;17(2):233-44.

YASSUDA M. LASCA V, NERI A. Meta-memória e Auto-eficácia: Um Estudo de Validação de Instrumentos de Pesquisa sobre Memória e Envelhecimento. Psicologia: Reflexão e crítica, 2005, 18 (1), pp.78-90. 